\title{
Differences in plasma TIMP-1 levels between healthy people and patients with rectal cancer stage II or III
}

\author{
Irena Oblak ${ }^{1}$, Franc Anderluh¹, Vaneja Velenik¹, Barbara Mozina², Janja Ocvirk², \\ Eva Ciric ${ }^{1}$, Natasa Hrovatic Podvrsnik ${ }^{1}$
}

\author{
${ }^{1}$ Department of Radiotherapy, ${ }^{2}$ Department of Laboratory Diagnostics, ${ }^{3}$ Department of Medical Oncology, Institute of \\ Oncology Ljubljana, Ljubljana, Slovenia
}

Received 9 June 2011

Accepted 3 July 2011

Disclosure: No potential conflicts of interest were disclosed.

Correspondence to: Assist. Prof. Oblak Irena, MD, PhD, Department of Radiotherapy, Institute of Oncology Ljubljana, Zaloška 2, 1000 Ljubljana, Slovenia. Phone: +386 15879 290; Fax: +386 15879 304; E-mail: ioblak@onko-i.si

\begin{abstract}
Background. The purpose of the study was to analyse whether the levels of the tissue inhibitor of matrix metalloproteinases-1 (TIMP-1) are higher in patients with rectal cancer as compared with healthy blood donors.

Patients and methods. Two hundred and seventeen patients ( 147 male, 70 female) with histologically confirmed non-metastatic rectal cancer (clinical stage II-III) and 45 healthy blood donors ( 15 male, 30 female) were included in analysis. Patient's mean age was 66 years (range: $34-87$ years) and healthy blood donor's mean age was 35 years (range: 18-64 years). Plasma TIMP-1 concentrations were measured with an enzyme-linked immunosorbent assay (ELISA) using commercially available TIMP-1 ELISA kit. Mann-Whitney-test for independent groups was used to assess the differences of plasma TIMP-1 levels and clinicopathological parameters. Two-sided tests were used and the differences at $\mathrm{P}<0.05$ were considered as statistically significant.

Results. Median patients TIMP-1 level was $180 \mathrm{ng} / \mathrm{mL}$ (range: $22-538 \mathrm{ng} / \mathrm{mL}$ ); the mean ( \pm SD) level was 193.7 (79.5) $\mathrm{ng} / \mathrm{mL}$. The median healthy blood donors TIMP-1 level was $112 \mathrm{ng} / \mathrm{mL}$ (range: $48-211 \mathrm{ng} / \mathrm{mL}$ ); the mean ( \pm SD) level was 115 (35.7) ng/mL. TIMP-1 levels in patients with rectal cancer were statistically significantly higher than TIMP-1 levels in healthy blood donors $(P<0.0001)$. Significant differences in TIMP-1 levels were not found comparing gender $(P=0.43)$, but in both groups TIMP-1 levels were increased with higher age ( $P=0.007)$.

Conclusions. Patients with rectal cancer had statistically significantly higher mean and median TIMP-1 level than healthy blood donors which is in accordance with the results published in other publications. These findings suggest possibility that plasma TIMP-1 levels could be used as new biological markers for early cancer detection.
\end{abstract}

Key words: TIMP-1; rectal cancer; healthy blood donors

\section{Introduction}

Tumour aggressiveness and metastatic potential, resulting in a poor prognosis, have been shown to be increased with the activation of matrix metalloproteinases (MMPs) and their tissue inhibitors (TIMPs). They play an important role in the process of degradation of the extracellular matrix and the basal membrane in relation to tumours invasiveness. The activity of the MMPs depends on the balance between the level of the active enzyme and its tissue inhibitor. ${ }^{1-4}$ The TIMP family consists of four members: TIMP-1, TIMP-2, TIMP-3 and
TIMP-4, which primary act as negative regulators of the degradation process of the extracellular matrix. Latest evidence suggests that they also participate in cell signalling by regulating cell growth, apoptosis, angiogenesis and genomic instability. $3,5,6$

Many researchers focused on clinical impact of TIMPs in cancer biology by rating tumour tissue TIMP expression or plasma circulating TIMP level. They found out that TIMPs can be useful diagnostic and prognostic markers like other new markers., ${ }^{3,6-8}$ Most of these studies evaluated TIMP-1 and they recognized it as an important marker for patient management and prognosis in several ma- 


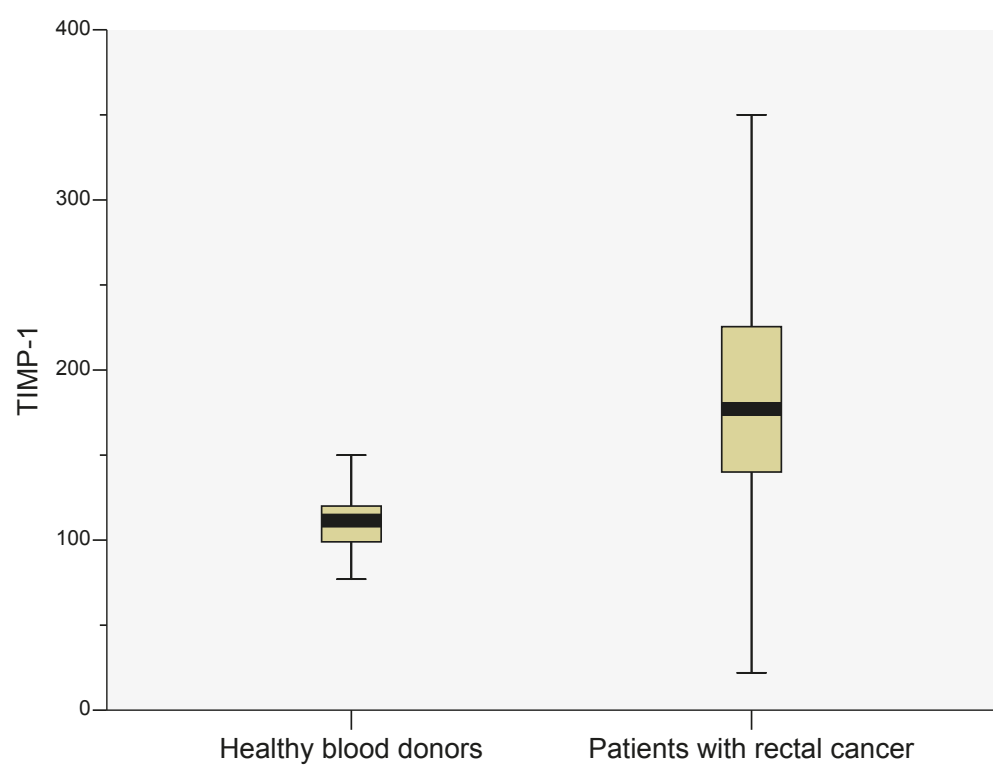

FIGURE 1. TIMP-1 levels (in $\mathrm{ng} / \mathrm{mL}$ ) in healthy blood donors and in patients.

lignancies such as colorectal, breast, lung, ovarian, bladder carcinoma ${ }^{3,6}$ and in several nonmalignant diseases such as asthma, diabetes, cardiovascular and autoimmune diseases. ${ }^{6}$

Elevated plasma TIMP -1 levels were reported to be significantly increased in colon cancer patients compared with healthy population, and they were also associated with advanced disease stage and poor prognosis., $3,5,6,9-12$ The aim of this study is to determine whether the TIMP-1 levels are higher in patients with rectal cancer as compared with healthy blood donors.

\section{Patients and methods}

\section{Patients}

Two hundred and seventeen patients (147 male, 70 female) with histologically confirmed non-metastatic rectal cancer (clinical stage II or III) and 45 healthy blood donors (15 male, 30 female) were included in analysis. Patient's mean age was 66 years (range: 34-87 years) and healthy blood donor's mean age was 35 years (range: $18-64$ years).

\section{Determination of plasma TIMP-1 level}

All blood samples were taken in the same laboratory, and processed and stored with the same procedures. The blood samples were collected in vacutainer EDTA tubes. After centrifugation at $1500 x g$ at $4^{\circ} \mathrm{C}$ for 10 minutes, plasma was separated and kept at $-80^{\circ} \mathrm{C}$ until analysis. Plasma TIMP-1 concentrations were measured with an enzymelinked immunosorbent assay (ELISA) using commercially available TIMP-1 ELISA kit, purchased from Oncogene Science, Cambridge (MA), USA. All procedures were performed according to the manufacturer's protocol in duplicate using diluted samples. Measurements differed by less than $10 \%$ and the mean value was calculated and used for statistical analysis. The inter-assay precision coefficients of variation of TIMP-1 ranged between 3.9\% and $8.8 \%$ at the different levels.

\section{Statistical analysis}

Statistical analysis was performed using personal computer and software statistical package SPSS, version 13 (SPSS Inc., USA). The main endpoint of the study was to determine if rectal cancer patients have higher TIMP-1 levels compared to healthy blood donors. Mann-Whitney-test for independent groups was used to assess the differences of plasma TIMP-1 levels and clinicopathological parameters. Two-sided tests were used and the differences at $\mathrm{P}<0.05$ were considered as statistically significant.

\section{Ethical consideration}

Prior to analysis, all patients and healthy blood donors received detailed oral and written information about the research and procedures, and they signed an informed consent for their plasma analysis for the purposes of the research. The trial was approved by the ethic committees of the Institute of Oncology, Ljubljana, Slovenia and by the National Medical Ethics Committee of Republic of Slovenia and was in agreement with the Declaration of Helsinki.

\section{Results}

\section{TIMP-1 levels}

Median patients TIMP-1 level was $180 \mathrm{ng} / \mathrm{mL}$ (range: $22-538 \mathrm{ng} / \mathrm{mL}$ ); the mean $( \pm S D)$ level was 193.7 (79.5) ng/mL. The median healthy blood donors TIMP-1 level was 112 ng/mL (range: 48-211 $\mathrm{ng} / \mathrm{mL})$; the mean $( \pm \mathrm{SD})$ level was 115 (35.7) ng/ $\mathrm{mL}$ (Figure 1). TIMP-1 levels in patients with rectal cancer were statistically significantly higher than TIMP-1 levels in healthy blood donors $(\mathrm{P}<0.0001)$. Significant differences in TIMP-1 levels were not found comparing gender $(\mathrm{P}=0.43)$, but in both groups TIMP-1 levels were increased with higher age $(\mathrm{P}=0.007)$ (Figures 2 and 3$)$. 


\section{Discussion}

TIMP-1 plays an important role in regulation of extracellular matrix remodelling, which is one of the processes involved in cancer growth and spread. Several authors reported that patients with malignant disease had significantly higher TIMP-1 levels in comparison with healthy population. ${ }^{5,9,11-14}$ In our study similar results were found. Patients with rectal cancer had statistically significant higher TIMP-1 levels in comparison with healthy blood donors $(\mathrm{P}<0.0001)$.

These findings also raise the idea that plasma TIMP-1 measurements could be useful in screening for malignant disease, such as rectal cancer in our case. Future randomised studies will demonstrate if the plasma TIMP-1 could be helpful for screening and detecting colorectal cancer beside digitorectal examination, faecal occult blood testing, recto-, sigmoido- and colonoscopy, DNA stool analysis and genetic testing. ${ }^{8,15}$ Screening has in oncology special value as patients with lower stages have better prognosis. ${ }^{16}$

Like other researchers ${ }^{3,12,14}$ we did not find significant differences in TIMP-1 levels and gender, but Holten-Andersen et al. ${ }^{11}$ demonstrated that the mean TIMP-1 levels were approximately $10 \%$ higher in males than in females. The reason for this they attributed to the fact that the male population has higher incidence of tromboembolic diseases, which result in higher release rate of TIMP-1 in plasma from activated platelets. ${ }^{11}$

In many studies (in ours as well) TIMP-1 level was found to be increased with age, $3,9,11,14,17$ although Tayebjee et al. in their study found an inverse relationship between TIMP-1 level and age. ${ }^{18}$

\section{Conclusions}

Patients with rectal cancer had statistically significantly higher mean and median TIMP-1 level than healthy blood donors which is in accordance with the results published in others publications. These findings suggest possibility that plasma TIMP-1 levels could be used as new biological markers for early cancer detection.

\section{References}

1. Pesta M, Topolcan O, Holubec L, Rupert K, Cerna M, Holubec L, et al. Clinicopathological assesment and quantitative estimation of the matrix metalloproteinases MMP-2 and MMP-7 and the inhibitors TIMP-1 and TIMP-2 in colorectal carcinoma tissue samples. Anticancer Res 2007; 27: 1863-8.

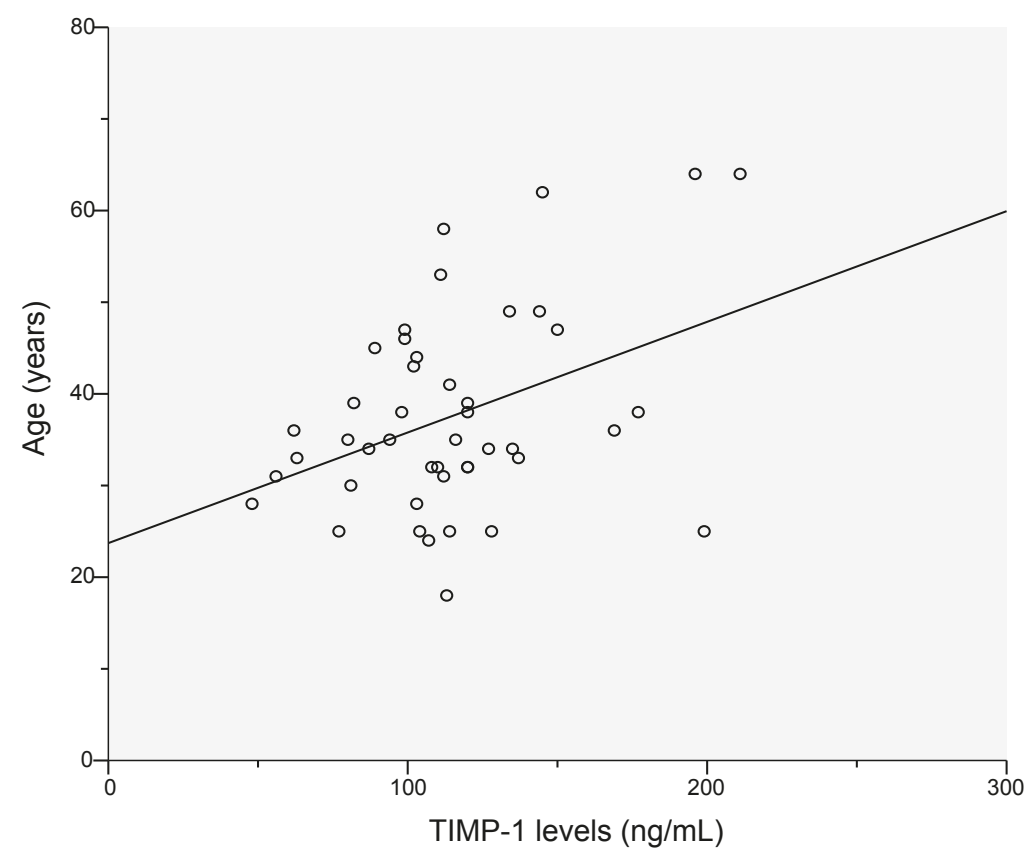

FIGURE 3. TIMP-1 levels and age in patients $(p=0.007)$.

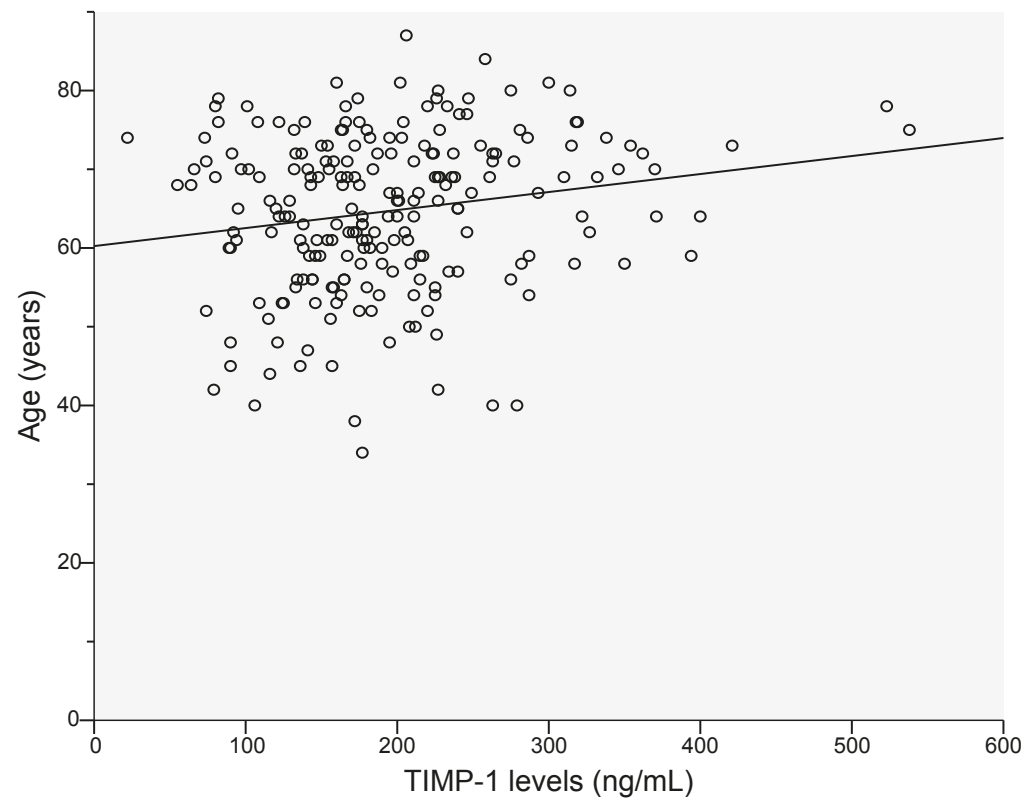

FIGURE 2. TIMP-1 levels and age in healthy blood donors ( $p=0.007)$.

2. Li M, Yamamoto H, Adachi $Y$, Maruyama $Y$, Shinomura $Y$. Role of matrix metaloproteinase-7 (matrilysin) in human cancer invasion, apoptosis, growth and angiogenesis. Exp Biol Med 2006; 231: 20-7.

3. Giaginis C, Nikiteas N, Margeli A, Tzanakis N, Rallis G, Kouraklis G, et al. Serum tissue inhibitor of metalloproteinase 1 and 2 (TIMP-1 and TIMP-2) levels in colorectal cancer patients: association with clinicopathological variables and patients survival. Int J Biol Markers 2009; 24: 245-52.

4. Alo-Aho R, Kahari VM. Colagenases in cancer. Biochemie 2005; 87: 273-86.

5. Sørensen NM, Sørensen IV, Würtz $S \varnothing$, Schrohl AS, Dowell B, Davis G, et al. Biology and potential clinical implications of tissue inhibitor of metalloproteinases-1 in colorectal cancer treatment. Scan J Gastroenterol 2008; 43: $774-86$. 
6. Frederiksen $\mathrm{CB}$, Lomholt $\mathrm{AF}$, Lottenburger $\mathrm{T}$, Davis $\mathrm{GJ}$, Dowell $\mathrm{BL}$, Blankenstein MA, et al. Assesment of the biological variation of plasma tissue inhibitor of metalloproteinase-1. Int J Biol Markers 2008; 23: 42-7.

7. Horvat M, Stabuc B. Microsatellite instability in colorectal cancer. Radiol Oncol 2011; 45: 75-81.

8. Yaolin Zhou Y, Boardman LA, Miller RC. Genetic testing for young-onset colorectal cancer: case report and evidence-based clinical guidelines. Radiol Oncol 2010; 44: 57-61.

9. Wass ET, Hendriks T, Lomme RM, Woblles T. Plasma levels of matrix metalloproteinase-2 and tissue inhibitor of metalloproteinase-1 correlate with disease stage and survival in colorectal cancer patients. Dis Colon Rectum 2005; 48: 700-10.

10. Kahlert C, Bandapalli OR, Schirmacher P, Weitz J, Brand K. Invasion frontspecific overexpression of tissue inhibitor of metalloproteinase-1 in liver metastases from colorectal cancer. Anticancer Res 2008; 28: 1459-66.

11. Holten-Andersen MN, Murphy G, Nielsen HJ, Pedersen AN, Christensen IJ, Høyer-Hansen G, et al. Quantitation of TIMP-1 in plasma of healthy blood donors and patients with advanced cancer. Br J Cancer 1999; 80: 495-503.

12. Hammer JH, Basse L, Svendsen MN, Werther K, Brüner N, Christensen IJ, et al. Impact of elective resection on plasma TIMP-1 in patients with colon cancer. Colorectal Dis 2006; 8: 168-72.

13. Holten-Andersen MN, Christensen IJ, Nielsen HJ, Stephens RW, Jensen V, Nielsen $\mathrm{OH}$, et al. Total levels of tissue inhibitor of metalloproteinases 1 in plasma yield high diagnostic sensitivity and specificity in patients with colon cancer. Clin Cancer Res 2002; 8: 156-64.

14. Holten-Andersen MN, Stephens RW, Nielsen HJ, Murphy G, Christensen IJ, Stetler-Stevenson W, et al. High preoperative plasma tissue inhibitor of metalloproteinase-1 levels are associated with short survival of patients with colorectal cancer. Clin Cancer Res 2000; 6: 4292-9.

15. Sofic A, Beslic S, Kocijancic I, Sehovic N. CT colonography in detection of colorectal carcinoma. Radiol Oncol 2010; 44: 19-23.

16. Oblak I, Petric P, Anderluh F, Velenik V, Hudej R, Fras AP. Anal cancer chemoirradiation with curative intent - a single institution experience. Neoplasma 2009; 56: 150-5

17. Ishida $H$, Murata $N$, Hayashi $Y$, Tada $M$, Hashimoto D. Serum levels of tissue inhibitor of metalloproteinases-1 (TIMP- 1) in colorectal cancer patients. Surg Today 2003; 33: 885-92.

18. Tayebjee MH, Lip GYH, Blann AD, MacFadyen RJ. Effects of age, gender, ethnicity, diurnal variations and exercise on circulating levels of matrix metalloproteinases (MMP)-2 and -9 , and their inhibitors, tissue inhibitors of matrix metalloproteinases (TIMP)-1 and -2. Thromb Res 2005; 115: 205-10. 